\title{
Article \\ Efficient and Robust Image Communication Techniques for 5G Applications in Smart Cities
}

\author{
Lavish Kansal $\left.^{1}{ }^{(}\right)$, Gurjot Singh Gaba ${ }^{1}\left(\mathbb{D}\right.$, Naveen Chilamkurti ${ }^{2}\left(\mathbb{D}\right.$ and Byung-Gyu Kim ${ }^{3, *(\mathbb{D}}$ \\ 1 School of Electronics \& Electrical Engineering, Lovely Professional University, Phagwara 144411, India; \\ lavish.kansal@lpu.co.in (L.K.); gurjot.17023@lpu.co.in (G.S.G.) \\ 2 Department of Computer Science and Computer Engineering, La Trobe University, \\ Melbourne, VIC 3086, Australia; n.chilamkurti@latrobe.edu.au \\ 3 Intelligent Vision Processing Laboratory (IVPL), Department of IT Engineering, \\ Sookmyung Women's University, Seoul 04310, Korea \\ * Correspondence: bg.kim@sookmyung.ac.kr
}

Citation: Kansal, L.; Gaba, G.S.; Chilamkurti, N.; Kim, B.-G. Efficient and Robust Image Communication Techniques for 5G Applications in Smart Cities. Energies 2021, 14, 3986. https://doi.org/10.3390/en14133986

Academic Editor:

William Holderbaum

Received: 6 June 2021

Accepted: 29 June 2021

Published: 2 July 2021

Publisher's Note: MDPI stays neutral with regard to jurisdictional claims in published maps and institutional affiliations.

Copyright: (c) 2021 by the authors. Licensee MDPI, Basel, Switzerland. This article is an open access article distributed under the terms and conditions of the Creative Commons Attribution (CC BY) license (https:/ / creativecommons.org/licenses/by/ $4.0 /)$.

\begin{abstract}
A wide range of multimedia applications must be supported by the modern fifth generation $(5 \mathrm{G})$ wireless communication systems for realizing the diverse applications in smart cities. The diverse applications such as real-time monitoring of roads, smart homes, smart industries, etc., for a sustainable smart city emphasizes a robust and efficient image transmission. In this paper, the influence of maximal ratio combining (MRC) on the reception of images with different orthogonal frequency division multiplexing (OFDM) versions is studied. The different OFDM versions considered here are the fast Fourier transform (FFT) based OFDM and discrete cosine transform (DCT) based OFDM. A comparison between diverse modulation levels for the images transmitted through different OFDM methodologies, along with variation in a number of receiving antennas for $M R C$, is proposed for additive white gaussian noise (AWGN) and Rayleigh fading channels. The diverse modulation levels used are binary phase shift keying (BPSK), quadrature phase shift keying (QPSK), 8-PSK, and 16-PSK. The parameters that are used to compare different versions of OFDM for MRC antenna configurations are signal-to-noise ratio (SNR) vs. bit error rate (BER) and peak signal-to-noise ratio (PSNR) at the receiver as an estimation parameter for the received image quality.
\end{abstract}

Keywords: OFDM; DCT; FFT; MRC; BER; PSNR

\section{Introduction}

The modern-day telecommunication systems implemented in smart cities require an elevated data rate and higher spectral efficiency to fulfil the requirements of multimedia application requirements of the users, which, in turn, is the main catalyst in the recent advancements. The rapidly growing popularity of $5 \mathrm{G}$ systems compared to the fourth generation $(4 \mathrm{G})$ systems is because the $5 \mathrm{G}$ systems promise data rates as high as $1 \mathrm{Gbps}$ [1]. In addition to the elevated data rates, features such as user mobility, security, and sustainability are also important in modern-day telecommunication systems implemented in smart cities. The 5G systems offer high data rates, but these high data rates are being offered by highly bandwidth efficient methodologies such as OFDM. The core ideology in the OFDM transmission is first to segregate the incoming data, and then the segregated data is being sent to the receiver end by utilizing the overlapping narrowband OFDM subcarriers. Since these narrowband subcarriers are orthogonal to each other, the presence of multipath fading is less effective in these subcarriers [2]. Many of the conventional systems, such as wireless interoperability for microwave access (WiMAX), Digital Audio Broadcasting (DAB), Terrestrial digital TV (DVB-T), long term evolution (LTE), and Wireless local area networks (LANs) make use of the OFDM methodology for transferring the information from one end to another end. However, in the quest of increasing the data capacity of the OFDM system, the BER experienced at the receiver side keeps on increasing due to the 
existence of inter-carrier interference (ICI) and inter-symbol interference (ISI) [2] Besides, elevated BER the OFDM system also experiences the problem of high PAPR, which occurs due to the non-linear operations of the power amplifiers installed at the transmitter side.

To mitigate such problems, various hybrid schemes are implemented in combination with OFDM systems. One of them is the use of various space diversity techniques, i.e., transmit diversity and receiver diversity. Multiple antennas are installed at the transmitter side to transmit diversity, whereas, in receiver diversity, the receiver side is being equipped with multiple antennas [3]. Diversity reception is the most widely used methodology utilized in advanced wireless communication systems like OFDM, WiMAX, Wideband code division multiple access (WCDMA), and multiple input multiple output (MIMO). One of the prime diversity reception techniques is diversity combining multiple antennas only at the receiver side. Diversity combining techniques are further classified into three categories, i.e., MRC, Equal Gain Combining (EGC), and Selection Combining (SC). MRC is the standout performer that performs much better than other combining schemes $[4,5]$. Image and Video transmission are a major application area of wireless communication systems. The concept of building a smart city is based on making efficient use of resources and transmission of real-time information from one place to another quickly in a very robust way. The paper's proposed methodology emphasizes efficient transmission of images from one place to another with minimum possible errors, making it most suitable for sustainable smart city applications like smart homes, smart industries, robotics and automation, smart roads, etc.

The remaining manuscript is organized as follows: Section 2 presents the state of the work, whereas Section 3 discusses the FFT-OFDM and DCT-OFDM system model. Section 4 provides the proposed system model and parameters. Section 5 highlights the significant results and comparative analysis. Section 6 exhibits the concluding remarks.

\section{Related Work}

In the preliminary work, the evaluation of three types of diversity combining schemes has been presented. The analysis has been carried out by pre-detection and post-detection merits and demerits [3]. The diversity technique based on maximal ratio combining exhibits an enhancement in signal-to-noise ratio over fading channels compared to the single branch receiving system. Direct relationship between performance improvement and the number of diversity branches combined was presented in literature [4]. The maximum output selection scheme performed better than maximum SNR regarding BER for M-ary modulation schemes over Rayleigh channel [5]. The utilization of spatial diversity provides a noteworthy enhancement in the system capacity over flay-Rayleigh and frequency selective channels with optimum combining/equalization being employed [6]. The influence of fading on the BER can be reduced by employing the diversity reception. The performance enhancement was reported for both correlated and non-correlated fading channels [7]. The comparison between optimum combining (OC) and MRC was presented for two co-channel interferers and a random number of interferers, respectively. The Monte Carlo simulation was used to assess the system behavior by BER [8]. The closed form BER performance analysis has been presented for the equal-gain combiner with 2 or 3 branches over Rayleigh fading channel [9]. The analysis of OC and MRC showed that the channel correlation of the interfering signal did not affect the BER performance, whereas the BER performance got severely affected once the channel correlation of the anticipated signal approaching unity [10]. The study of MRC for wireless communication showed that the SNR is varying by the cross correlation between the channel vectors [11]. The use of hybrid transmit diversity was also being demonstrated by employing the selection diversity and maximal ratio combining jointly. The hybrid combination offered an improved BER performance in comparison to maximal ratio combining working alone [12-18]. The performance examination for multiple input multiple output (MIMO) MRC is demonstrated over the rician fading channel. On studying the result, it was very much evident from the simulation results that the behavior of single input multiple output (SISO) systems was equivalent 
to multiple input single output (MISO) system provide that the total antenna elements are fixed [19]. The BER of MRC and EGC was analyzed over gamma fading channels by considering the independent gamma fading on the diversity branches [20]. DCT offers an efficient alternative to DFT for the implementation of multicarrier transceivers. It can eliminate the ICI and ISI at a lower complexity without any guard interval [21]. The overall system performance of MRC with arbitrary fading parameters was analyzed over the flat-Nakagami-m fading channel [22]. The BER investigation for the DCT OFDM system and carrier frequency offset (CFO) on AWGN channel has been presented. The simulation analysis undoubtedly illustrates that the BER characterization of DCT-OFDM is reliable than the BER characterization of DFT-OFDM [23,24]. The OFDM system based on the DCT outclasses the DFT-OFDM system by providing enhanced BER performance over the multipath fading channel having time-varying characteristics. The enhancement was nearly $5.5 \mathrm{~dB}$ regarding the signal-to-noise ratio [25]. The OFDM system based on DFT, DCT, and discrete wavelet transform (DWT) has been analyzed over AWGN channel with BPSK and QPSK are the modulation schemes being used. The BER analysis showed that DWT-OFDM outperforms the DFT-OFDM and DCT-OFDM. Additionally, the DCT-OFDM still provides better BER characterization in contrast to DFT-OFDM [26]. The analysis of MIMO systems by BER characterization was presented for OC and MRC in the existence of co-channel interference. The modulation scheme used for the study of system characterization is quadrature amplitude modulation (QAM) [27]. The BER study of wireless MIMO systems employing the Alamouti's Scheme and MRC technique was presented [28]. The simulation outcome depicts a substantial enhancement in the BER performance over small values of SNR. The transmission of images using OFDM systems over the wireless channels was also being analyzed. The image quality was analyzed by DFT-OFDM, DCT-OFDM, and DWT-OFDM systems [29]. The efficient image transfer using OFDM over wireless channels with reduced PAPR is proposed [30]. Various discrete transforms were used in OFDM systems such as DFT, DCT, and DWT. The comparison shows that DCT/DWT in place of DFT for conventional OFDM systems significantly reduces the PAPR thereby improving the image quality at the receiver end [31-37]. Table 1 presents a comparative analysis of work presented in literature and the proposed methodology.

Table 1. Comparative analysis of work presented in literature and the proposed methodology.

\begin{tabular}{c}
\hline Article \\
\hline BER Performance Analysis of Image \\
Transmission Using OFDM Technique in \\
Different Channel Conditions Using \\
Various Modulation Techniques, \\
Computational Intelligence in Data Mining.
\end{tabular}

A two layer chaotic encryption scheme of secure image transmission for DCT precoded OFDM-VLC transmission.

Performance assessment of pre-coded OFDM using discrete cosine-based DOST transform.

\section{Research Outcome}

This paper discussed the BER performance analysis of Image Transmission over conventional OFDM system using various modulation technique. The present manuscript presents the BER performance analysis of MIMO-OFDM system, which makes it more appropriate for the current application requirements of $5 \mathrm{G}$ systems.

In this paper, a two-layer image encryption scheme for a discrete cosine transform (DCT) pre-coded orthogonal frequency division multiplexing (OFDM) visible light communication (VLC) system is proposed. The present manuscript also utilized the concept of DCT pre-coded OFDM system, but it has been analyzed over radio channel and by employing multiple antennas at receiver side. Additionally, in addition to using BER performance as comparison metric, PSNR vs. SNR and SSIM are also used to present the reliability of the proposed methodology.

In this research article an innovative precoding method has been proposed based on Discrete Cosine-based DOST (DCST) to lessen the High PAPR values and BER in OFDM systems. A similar concept of precoding is being utilized in the present manuscript with the use of DCT instead of DCST transform. Moreover, the analysis is carried out by utilizing the image transmission methodology instead of generic data sequences.

This paper compares the quality of diffusion-based and permutation-based encrypted image transmission using orthogonal frequency division multiplexing (OFDM) over wireless fading channel. In the present manuscript, no encryption is being utilized. The comparison of the quality of transmitted and received image is
Optimized OFDM transmission of encrypted image over fading channel. carried out with the help of SSIM metric. 
Based on the literature review, it is evident that to provide an efficient and robust information transmission as required in $5 \mathrm{G}$ application. We need to utilize the hybrid combination of multi-carrier technology like OFDM and multiple antenna methodologies. However, the literature also presents that the convention Fourier transform-based OFDM suffers from ISI and ICI's impact, which can be reduced by replacing the Fourier transform with other transforms. The efficient and robust transmission of images and other multimedia information is critical for $5 \mathrm{G}$ applications, so it is important to assess the proposed system performance for image transmission over diverse channels i.e., AWGN and Rayleigh channel. The system's robustness can be easily carried out by using BER analysis, and the received image quality can be easily accessed by analyzing the PSNR and structural similarity index (SSIM) variations.

\section{Our Contribution}

Based on the study of the research work reported in the literature survey, the following research gaps are addressed to realize the goal of efficient multimedia telecommunication methodology in a sustainable smart city:

- DCT transform is being utilized to improve the BER performance in place of Fourier transform in the OFDM system for efficient transmission of the image.

- A hybrid combination of MIMO-OFDM system is presented as a valid alternative to the conventional OFDM system for image transmission.

- The numbers of antennas are varied at the receiver side to access the MIMO-OFDM system's performance and incorporate diverse transforms for multimedia applications.

\section{Model Description}

\subsection{FFT-OFDM System Model}

The incoming data stream is first encoded by using the source coding techniques to represent the data efficiently. The source encoded data is then sent to the channel encoding block with additional bits to the incoming data to ensure that the received data's errors can be detected and corrected at the receiver end. The encoded data is then modulated using the M-PSK modulation; the modulated data is then forwarded to the inverse fast Fourier transform (IFFT) block. The IFFT block maps the modulated data onto the orthogonal subcarriers, as presented in Figure 1. The $\mathrm{n}^{\text {th }}$ element of discrete-time complex OFDM symbol can be written as:

$$
\mathrm{X}_{\mathrm{n}}=\frac{1}{\mathrm{~N}} \sum_{\mathrm{k}=0}^{\mathrm{N}-1} \mathrm{X}_{\mathrm{k}} \mathrm{e}^{\mathrm{j} \frac{2 \pi \mathrm{kn}}{\mathrm{N}}} 0 \leq \mathrm{n} \leq \mathrm{N}-1,0 \leq \mathrm{k} \leq \mathrm{N}-1
$$

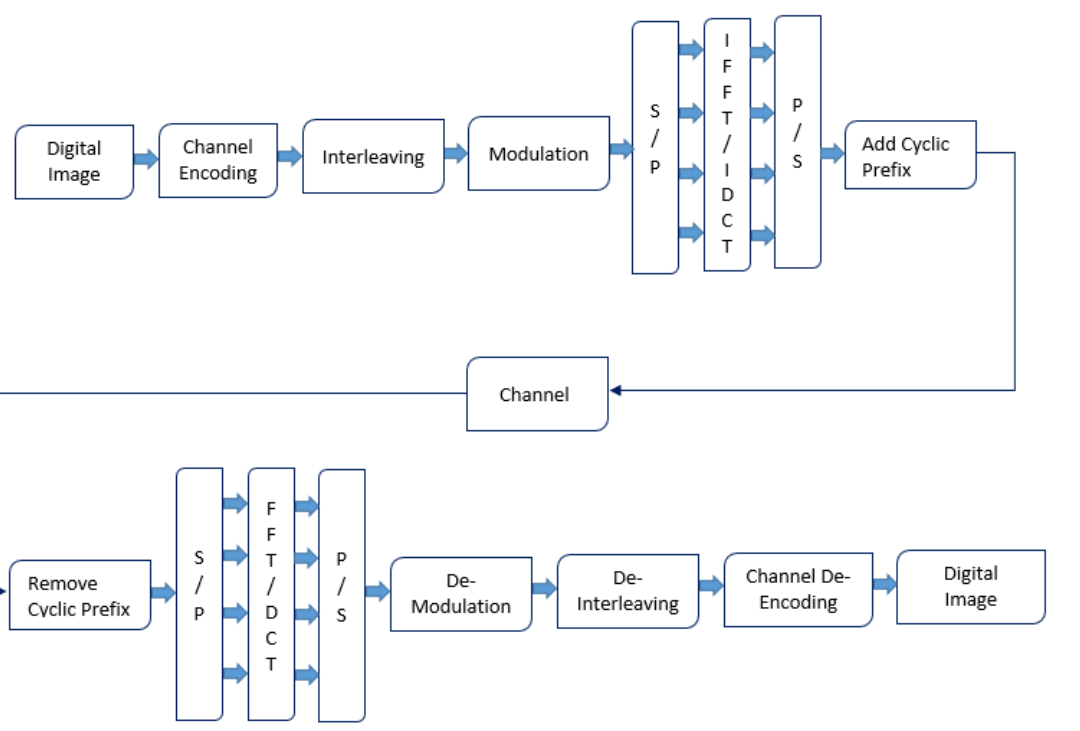

Figure 1. Block Diagram of OFDM System. 
Here, Equation (1) represents the discrete-time complex OFDM symbol. N represents the number of samples, $X_{k}$ represents the input signal, and $X_{n}$ represents the OFDM symbols obtained after the Fourier transform. After the orthogonal mapping of the data, the addition of guard interval is being done, ensuring that the received data would be free from the ISI effects. At the receiver end, the entire process is reversed to extract the original information.

\subsection{DCT-OFDM System Model}

The DCT based OFDM system is analogous to FFT based OFDM system, but the IFFT and FFT modules are swapped by inverse discrete cosine transform (IDCT) and DCT modules. DCT utilizes the real arithmetic instead of the complex arithmetic being utilized in the FFT, which results in the reduction of signal processing complication as well as in-phase/quadrature imbalance. DCT reduces the ISI and also makes efficient utilization of transmitted samples close to zero by its outstanding spectral energy compaction property. The transmitted signal for the DCT based OFDM is depicted in Equation (2). $X_{k}$ represents the input signal, and $X_{n}$ represents the $\mathrm{n}^{\text {th }}$ OFDM symbols obtained after DCT transform.

$$
\begin{aligned}
& X_{n}=\sqrt{\frac{2}{N}} \sum_{k=0}^{N-1} X_{k} \beta(k) \cos \left(\frac{\pi k(2 n+1)}{2 N}\right) 0 \leq n \leq N-1,0 \leq k \leq N-1 \\
& \beta(\mathrm{k})=\left\{\begin{array}{c}
\frac{1}{\sqrt{2}} k=0 \\
1 k=1,2, \ldots N-1
\end{array}\right\}
\end{aligned}
$$

\subsection{Maximal Ratio Combining (MRC)}

In MRC diversity scheme, the output is obtained by summation of all branches that are weighted by different weight parameters, so the $\alpha_{\mathrm{i}} \mathrm{s}$ in Figure 2 are all zero. Subsequently, the signals are co-phased, $\alpha_{i}=\alpha_{i} e^{-j \theta_{i}}$, where $\theta_{i}$ is the phase of the incoming signal in the ith branch. The envelope of the combiner output is presented in Equation (3). It represents that the received signal is the submission of the input signal coming from different branches, i.e., $r_{i}$ multiplied by different weight parameters.

$$
\mathrm{r}=\sum_{\mathrm{i}=1}^{\mathrm{M}} \mathrm{a}_{\mathrm{i}} \mathrm{r}_{\mathrm{i}}
$$

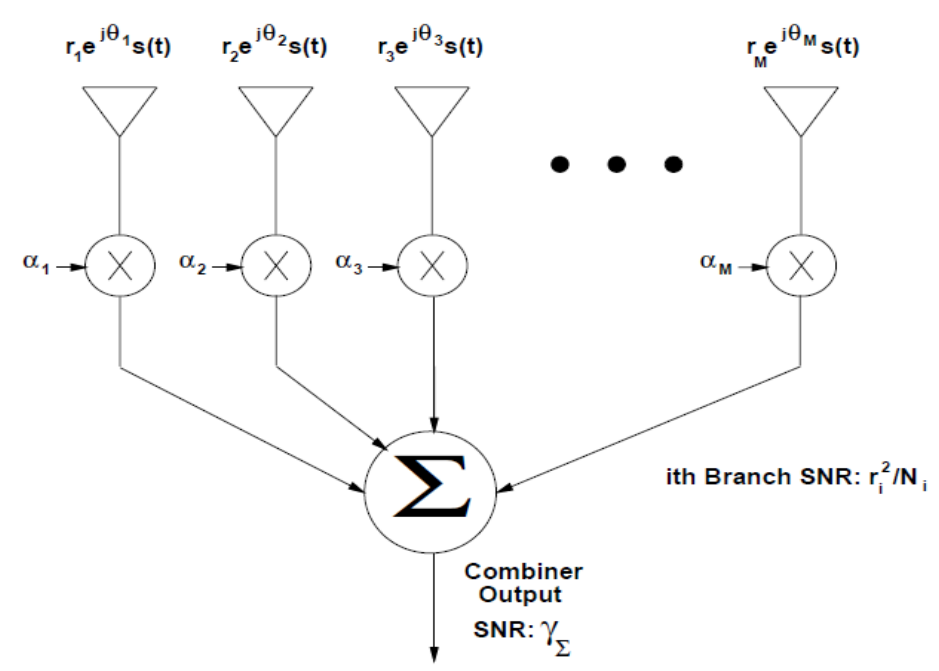

Figure 2. Linear Combiner. 
Supposing the same Noise Power Spectral Density (PSD) $\mathrm{N}_{0}$ in each branch yields a total noise PSD. $\mathrm{N}_{\text {tot }}$ at the combiner output of and is depicted in Equation (4).

$$
\mathrm{N}_{\text {tot }}=\sum_{\mathrm{i}=1}^{\mathrm{M}} \mathrm{a}_{\mathrm{i}}^{2} \mathrm{~N}_{0}
$$

$\mathrm{N}_{\text {tot }}$ represents the total noise power at the receiver side. It is very evident from Equation (4) that the total noise power also depends upon the weight parameters. The weight parameters are decided to boost the signal that contains the information components and attenuate the signal, which contains noise components. Therefore, the output SNR of the combiner is presented in Equation (5). It depicts that the SNR is the ratio of the weighted received signal and weighted noise signal of all the branches. The weight parameters must be optimally defined to have maximum SNR.

$$
\gamma_{\Sigma}=\frac{\mathrm{r}^{2}}{\mathrm{~N}_{\text {tot }}}=\frac{1}{\mathrm{~N}_{0}} \frac{\left(\sum_{\mathrm{i}=1}^{\mathrm{M}} \mathrm{a}_{\mathrm{i}} \mathrm{r}_{\mathrm{i}}\right)^{2}}{\sum_{\mathrm{i}=1}^{\mathrm{M}} \mathrm{a}_{\mathrm{i}}^{2}}
$$

To maximize the $\gamma_{\Sigma}$, the optimum selection of $\alpha_{\mathrm{i}} \mathrm{s}$ is required. The optimum selection should be done so that the branches having higher SNR must be weighted more compared to branches having lower SNR values. This weighting strategy will result in making the weights $a_{i}^{2}$ proportional to the branch SNRs $r_{i}^{2} / N_{0}$. The values of $\alpha_{i} s$ that will maximize the $\gamma_{\Sigma}$ can be obtained by taking the partial derivatives of Equation (6) or using the Swartz inequality. Unraveling for the optimal weights yields $a_{i}^{2}=r_{i}^{2} / N_{0}$, and the resultant combiner SNR becomes:

$$
\gamma_{\Sigma}=\sum_{\mathrm{i}=1}^{\mathrm{M}} \mathrm{r}_{\mathrm{i}}^{2} / \mathrm{N}_{0}=\sum_{\mathrm{i}=1}^{\mathrm{M}} \gamma_{\mathrm{i}}
$$

\section{Proposed System Model and Parameters}

In this work MRC diversity combining scheme is being used along with OFDM system to investigate the quality of image reception along with the use of DCT in place of FFT for OFDM system as presented in Figure 3. MRC scheme is being used in conjunction with OFDM system to investigate the image quality enhancement (PSNR and BER) for both the FFT and DCT transform. SNR vs. BER and SNR vs. PSNR are two parameters in which the system's performance is investigated by varying the number of receiving antennas and transforms.

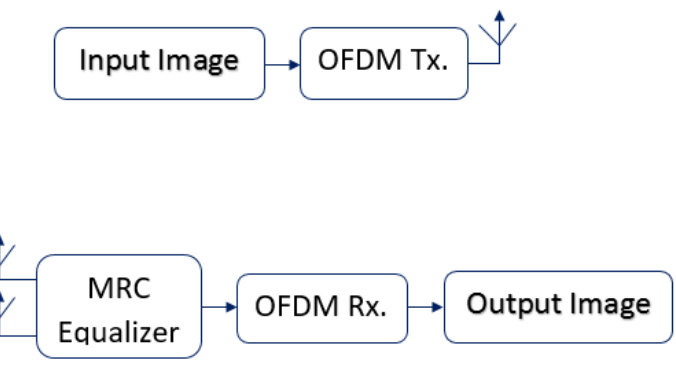

Figure 3. Block Diagram of OFDM with MRC.

PSNR between the original image and the demodulated image is utilized to evaluate the quality of the received image, which is defined in Equation (7).

$$
\mathrm{PSNR}=10 \log _{10}\left(\frac{255^{2}}{\mathrm{MSE}}\right)
$$

where the mean square error (MSE) is defined as:

$$
\operatorname{MSE}=\frac{1}{N^{2}} \sum_{i=1}^{N} \sum_{j=1}^{N}[f(i, j)-\hat{f}(i, j)]^{2}
$$


where $f(i, j)$ is the original image of dimensions $N \times N$ and $\hat{f}(i, j)$ is the demodulated image. MSE, as depicted in Equation (8), tells us about the quality of reconstructions of the image at the receiver side. If MSE is higher, than it is clear that the image is not reconstructed at the receiver side correctly, and PSNR will be less. Similarly, if the MSE is lower, it implies that the image reconstruction is near the optimum level, and the PSNR value will be high.

\section{Result Analysis and Discussion}

The evaluation of FFT-OFDM and DCT-OFDM augmented by MRC receiver diversity scheme is presented for image transmission. In Figures 4-7, a visual representation of image quality is presented for FFT-OFDM and DCT-OFDM using MRC (1 Tx and 1, 2, 3 $\mathrm{Rx}$ ) under AWGN and Rayleigh channel. The comparison is done for diverse modulation schemes, and the representation is presented for various SNR values. It is apparent from the representations that as the modulation level keeps growing, the image quality is degrading at a particular SNR value. However, if we increase the SNR from $5 \mathrm{~dB} 15 \mathrm{~dB}$, the improvement in the received image quality can be seen. It is also quite evident from the comparison that it can also be detected as we raise the number of receiving antennas from 1 to 2, and in 3 the image quality enhances in both the FFT-OFDM and DCT-OFDM. This is because the receiver diversity provided by MRC schemes minimizes the effect of the channel environment on the signal. The use of multiple receivers at the receiver side provides a better quality of data reception than a single antenna at the receiver end. The comparison of Figures 4 and 5 also depicts that the received image quality is better in case of DCT-OFDM in comparison to FFT-OFDM. However, the image quality got affected under the influence of Rayleigh fading channel.

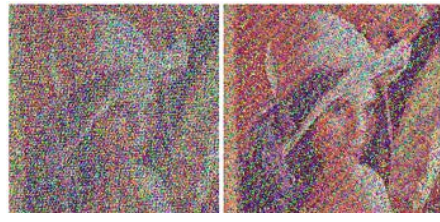

(a) $\mathrm{nTx}=1$ and $\mathrm{nRx}=1$

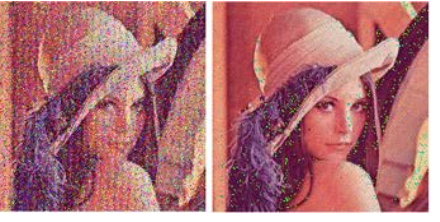

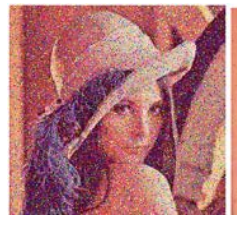

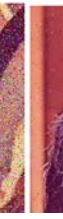

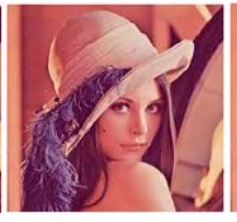

(b) $\mathrm{nTx}=1$ and $\mathrm{nRx}=2$

Figure 4. Received Image with FFT-OFDM over a AWGN channel, Modulated with QPSK and at SNR =0, 5, 10, 15 dB from $\mathrm{L}=>\mathrm{R}$ with $\operatorname{MRC}(\mathrm{nTx}=1$ and $\mathrm{nRx}=1$ and 2$)$.
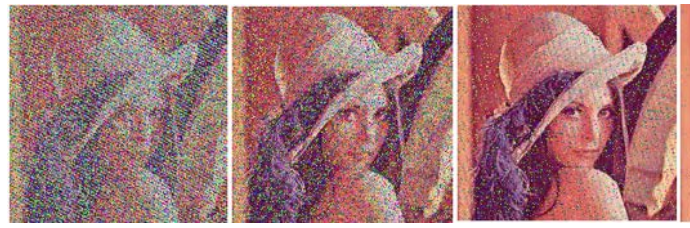

(a) $\mathrm{nTx}=1$ and $\mathrm{nRx}=1$
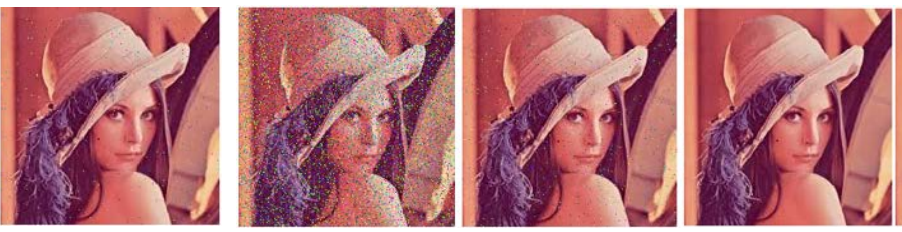

(b) $\mathrm{nTx}=1$ and $\mathrm{nRx}=2$

Figure 5. Received Image with DCT-OFDM over a AWGN channel, Modulated with QPSK and at SNR = 0, 5, 10, $15 \mathrm{~dB}$ from $L=>R$ with MRC (nTx $=1$ and $n R x=1$ and 2$)$.
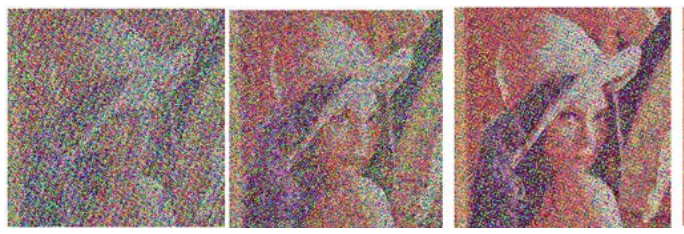

(a) $\mathrm{nTx}=1$ and $\mathrm{nRx}=1$
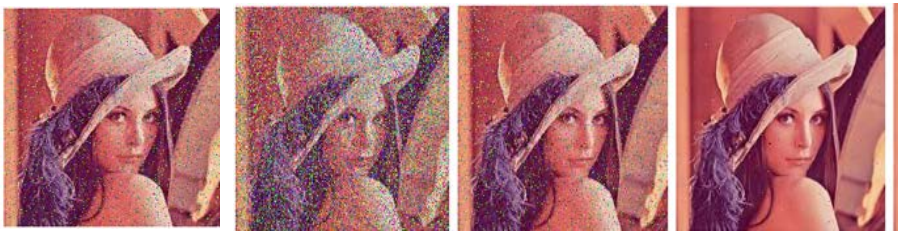

(b) $n T x=1$ and $n R x=2$

Figure 6. Received Image with FFT-OFDM over a Rayleigh channel, Modulated with QPSK and at SNR = 0, 5, 10, $15 \mathrm{~dB}$ from $L=>R$ with MRC ( $\mathrm{nTx}=1$ and $n R x=1$ and 2). 

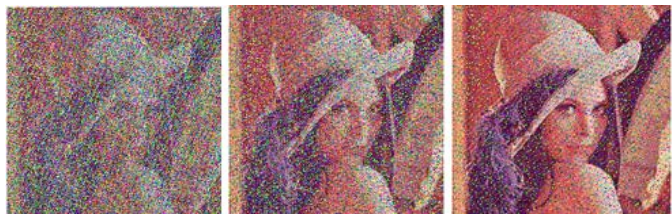

(a) $\mathrm{nTx}=1$ and $\mathrm{nRx}=1$
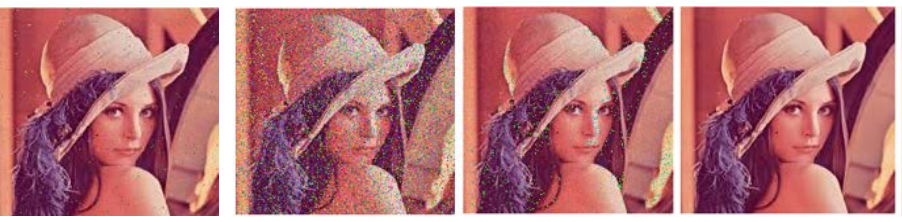

(b) $\mathrm{nTx}=1$ and $\mathrm{nRx}=2$

Figure 7. Received Image with DCT-OFDM over a Rayleigh channel, Modulated with QPSK and at SNR = 0, 5, 10, $15 \mathrm{~dB}$ from $L=>R$ with MRC (nTx $=1$ and $n R x=1$ and 2$)$.

Tables 2-5 illustrate the variations of the PSNR with SNR for both FFT-OFDM and DCT-OFDM (with and without MRC) over AGN channel, and Tables 6-9 illustrates the variations of the PSNR with SNR for both FFT-OFDM and DCT-OFDM (with and without MRC) over Rayleigh fading channel employing diverse modulation schemes for the RGB Lena image, similar results will also be there for other images. It is very much clear from the observations of Table 2 that there is a significant increase in the PSNR values for both FFT-OFDM and DCT-OFDM on employing the MRC schemes with $1 \mathrm{Tx}$ and $2 \mathrm{Rx}$ antennas. In the case of BPSK at SNR $=0 \mathrm{~dB}$, the PSNR value is $9.33109 \mathrm{~dB}$, and it is increased up to $15.8195 \mathrm{~dB}$ for FFT-OFDM. Similar inference can be made out by observing the values for DCT-OFDM for BPSK at SNR $=0 \mathrm{~dB}$, in which the PSNR value increases up to $21.1769 \mathrm{~dB}$ from $10.8239 \mathrm{~dB}$ on employing the MRC scheme with $1 \mathrm{Tx}$ and $2 \mathrm{Rx}$ antennas. Further, it can be observed that PSNR also enhances as we switch from FFT-OFDM to DCT-OFDM for all SNR values and both with MRC and without MRC schemes. Similar observations can be made from Tables 3-9 that the PSNR value enhances as we switch from FFT-OFDM to DCT-OFDM. Additionally, the PSNR value enhances on employing the MRC scheme with $1 \mathrm{Tx}$ and $2 \mathrm{Rx}$ antennas. This increase in PSNR values results in significant improvement in the quality of the image at the receiver end. Moreover, the PSNR decreases under the influence of Rayleigh fading channel as presented in Tables 6-9.

Table 2. PSNR values for FFT-OFDM and DCT-OFDM for BPSK with MRC (nTx = 1 and $n R x=1,2)$ over AWGN Channel.

\begin{tabular}{ccccc}
\hline \multirow{2}{*}{ SNR } & \multicolumn{2}{c}{ FFT-OFDM } & \multicolumn{2}{c}{ DCT-OFDM } \\
\cline { 2 - 5 } & $\mathbf{1}$ Tx and 1 Rx & $\mathbf{1 ~ T x}$ and 2 Rx & 1 Tx and 1 Rx & 1 Tx and 2 Rx \\
\hline $\mathbf{0 ~ d B}$ & 9.33109 & 15.8195 & 10.8239 & 21.1769 \\
\hline $\mathbf{5} \mathbf{~ d B}$ & 11.1558 & 27.0993 & 12.1698 & 42.7428 \\
\hline $\mathbf{1 0} \mathbf{~ d B}$ & 14.3648 & 58.0264 & 19.2917 & 60.2544 \\
\hline $\mathbf{1 5} \mathbf{~ d B}$ & 22.5041 & 69.4254 & 36.3608 & 73.5611 \\
\hline
\end{tabular}

Table 3. PSNR values for FFT-OFDM and DCT-OFDM for QPSK with MRC $(\mathrm{nTx}=1$ and $\mathrm{nRx}=1,2)$ over AWGN Channel.

\begin{tabular}{ccccc}
\hline \multirow{2}{*}{ SNR } & \multicolumn{2}{c}{ FFT-OFDM } & \multicolumn{2}{c}{ DCT-OFDM } \\
\cline { 2 - 5 } & $\mathbf{1}$ Tx and 1 Rx & $\mathbf{1 ~ T x}$ and 2 Rx & 1 Tx and 1 Rx & 1 Tx and 2 Rx \\
\hline $\mathbf{0 ~ d B}$ & 9.9119 & 16.0626 & 10.4111 & 21.2002 \\
\hline $\mathbf{5} \mathbf{~ d B}$ & 11.5252 & 26.9213 & 12.8423 & 44.3806 \\
\hline $\mathbf{1 0} \mathbf{~ d B}$ & 13.0074 & 59.5812 & 17.4532 & 62.5781 \\
\hline $\mathbf{1 5} \mathbf{~ d B}$ & 23.8034 & 70.2641 & 29.1656 & 76.5459 \\
\hline
\end{tabular}


Table 4. PSNR values for FFT-OFDM and DCT-OFDM for 8-PSK with MRC $(\mathrm{nTx}=1$ and $\mathrm{nRx}=1,2)$ over AWGN Channel.

\begin{tabular}{ccccc}
\hline \multirow{2}{*}{ SNR } & \multicolumn{2}{c}{ FFT-OFDM } & \multicolumn{2}{c}{ DCT-OFDM } \\
\cline { 2 - 5 } & $\mathbf{1}$ Tx and 1 Rx & $\mathbf{1 ~ T x}$ and 2 Rx & 1 Tx and 1 Rx & 1 Tx and 2 Rx \\
\hline $\mathbf{0 ~ d B}$ & 9.2832 & 12.5852 & 9.5323 & 13.5347 \\
\hline $\mathbf{5} \mathbf{~ d B}$ & 10.309 & 18.1539 & 10.616 & 20.5674 \\
\hline $\mathbf{1 0} \mathbf{~ d B}$ & 12.3526 & 32.8267 & 12.5531 & 42.1449 \\
\hline $\mathbf{1 5} \mathbf{~ d B}$ & 16.4483 & 59.4964 & 16.747 & 64.5412 \\
\hline
\end{tabular}

Table 5. PSNR values for FFT-OFDM and DCT-OFDM for 16-PSK with MRC (nTx = 1 and $n R x=1,2)$ over AWGN Channel.

\begin{tabular}{ccccc}
\hline \multirow{2}{*}{ SNR } & \multicolumn{2}{c}{ FFT-OFDM } & \multicolumn{2}{c}{ DCT-OFDM } \\
\cline { 2 - 5 } & $\mathbf{1}$ Tx and 1 Rx & $\mathbf{1 ~ T x}$ and 2 Rx & 1 Tx and 1 Rx & 1 Tx and 2 Rx \\
\hline $\mathbf{0 ~ d B}$ & 10.944 & 18.0643 & 11.688 & 20.7696 \\
\hline $\mathbf{5 ~ d B}$ & 11.7248 & 22.4797 & 13.3363 & 25.7564 \\
\hline $\mathbf{1 0} \mathbf{~ d B}$ & 16.9373 & 28.995 & 20.5709 & 36.2319 \\
\hline $\mathbf{1 5} \mathbf{~ d B}$ & 21.3388 & 45.3092 & 24.427 & 70.0577 \\
\hline
\end{tabular}

Table 6. PSNR values for FFT-OFDM and DCT-OFDM for BPSK with MRC (nTx $=1$ and $n R x=1,2)$ over Rayleigh Channel.

\begin{tabular}{ccccc}
\hline \multirow{2}{*}{ SNR } & \multicolumn{2}{c}{ FFT-OFDM } & \multicolumn{2}{c}{ DCT-OFDM } \\
\cline { 2 - 5 } & $\mathbf{1}$ Tx and 1 Rx & $\mathbf{1 ~ T x}$ and 2 Rx & 1 Tx and 1 Rx & 1 Tx and 2 Rx \\
\hline $\mathbf{0 ~ d B}$ & 8.5157 & 12.78663 & 10.1498 & 16.5133 \\
\hline $\mathbf{5} \mathbf{~ d B}$ & 10.1206 & 18.3998 & 11.7852 & 26.032 \\
\hline $\mathbf{1 0} \mathbf{~ d B}$ & 12.9643 & 34.54 & 15.559 & 56.4358 \\
\hline $\mathbf{1 5} \mathbf{~ d B}$ & 18.8008 & 54.3651 & 22.734 & 72.1541 \\
\hline
\end{tabular}

Table 7. PSNR values for FFT-OFDM and DCT-OFDM for QPSK with MRC $(\mathrm{nTx}=1$ and $\mathrm{nRx}=1,2)$ over Rayleigh Channel.

\begin{tabular}{ccccc}
\hline \multirow{2}{*}{ SNR } & \multicolumn{2}{c}{ FFT-OFDM } & \multicolumn{2}{c}{ DCT-OFDM } \\
\cline { 2 - 5 } & $\mathbf{1}$ Tx and 1 Rx & $\mathbf{1 ~ T x}$ and 2 Rx & 1 Tx and 1 Rx & 1 Tx and 2 Rx \\
\hline $\mathbf{0 ~ d B}$ & 9.6355 & 12.8861 & 9.9624 & 15.7346 \\
\hline $\mathbf{5} \mathbf{~ B B}$ & 10.5961 & 18.7141 & 12.1718 & 26.1021 \\
\hline $\mathbf{1 0} \mathbf{~ d B}$ & 19.331 & 34.0961 & 15.009 & 61.4828 \\
\hline $\mathbf{1 5} \mathbf{~ d B}$ & 19.037 & 53.1652 & 27.8149 & 76.5221 \\
\hline
\end{tabular}

Table 8. PSNR values for FFT-OFDM and DCT-OFDM for 8-PSK with MRC $(\mathrm{nTx}=1$ and $\mathrm{nRx}=1,2)$ over Rayleigh Channel.

\begin{tabular}{ccccc}
\hline \multirow{2}{*}{ SNR } & \multicolumn{2}{c}{ FFT-OFDM } & \multicolumn{2}{c}{ DCT-OFDM } \\
\cline { 2 - 5 } & $\mathbf{1}$ Tx and 1 Rx & $\mathbf{1 ~ T x}$ and 2 Rx & $\mathbf{1}$ Tx and 1 Rx & $\mathbf{1 ~ T x}$ and 2 Rx \\
\hline $\mathbf{0 ~ d B}$ & 9.2219 & 10.9362 & 9.228 & 11.4612 \\
\hline $\mathbf{5} \mathbf{~ d B}$ & 9.8334 & 13.8928 & 10.0164 & 15.2007 \\
\hline $\mathbf{1 0} \mathbf{~ d B}$ & 11.2669 & 21.5332 & 11.4974 & 25.1824 \\
\hline $\mathbf{1 5} \mathbf{~ d B}$ & 14.2133 & 43.1082 & 15.151 & 49.9177 \\
\hline
\end{tabular}


Table 9. PSNR values for FFT-OFDM and DCT-OFDM for 16-PSK with MRC (nTx $=1$ and $n R x=1,2)$ over Rayleigh Channel.

\begin{tabular}{ccccc}
\hline \multirow{2}{*}{ SNR } & \multicolumn{2}{c}{ FFT-OFDM } & \multicolumn{2}{c}{ DCT-OFDM } \\
\cline { 2 - 5 } & $\mathbf{1}$ Tx and 1 Rx & $\mathbf{1 ~ T x}$ and 2 Rx & 1 Tx and 1 Rx & 1 Tx and 2 Rx \\
\hline $\mathbf{0 ~ d B}$ & 10.4532 & 15.135 & 10.9457 & 17.853 \\
\hline $\mathbf{5} \mathbf{~ d B}$ & 11.7834 & 19.6491 & 13.9493 & 22.3086 \\
\hline $\mathbf{1 0} \mathbf{~ d B}$ & 14.6304 & 24.1127 & 18.4043 & 28.286 \\
\hline $\mathbf{1 5} \mathbf{~ d B}$ & 20.1877 & 32.447 & 22.2489 & 43.1996 \\
\hline
\end{tabular}

The same analysis has also been carried out using different images, as shown in Figures 8-11, and the outcome is similar to the previous outcome as depicted in Figures 4-7.

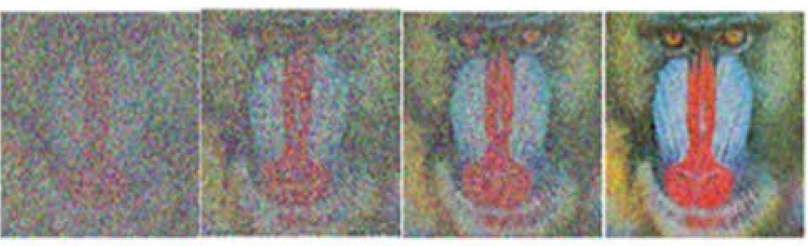

(a) $\mathrm{nTx}=1$ and $\mathrm{nRx}=1$
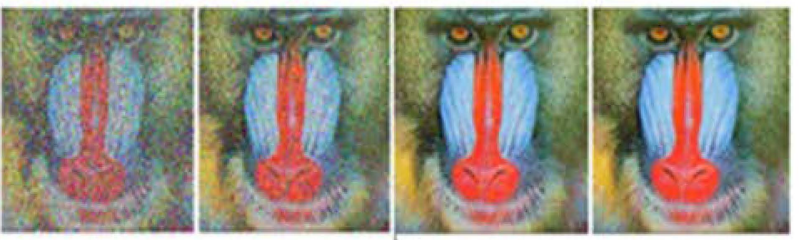

(b) $n T x=1$ and $n R x=2$.

Figure 8. Received Image with FFT-OFDM over a Rayleigh channel, Modulated with QPSK at Eb/No = 0, 5, 10, 15 dB from left to right with $\mathrm{MRC}(\mathrm{nTx}=1$ and $\mathrm{nRx}=1$ and 2$)$.
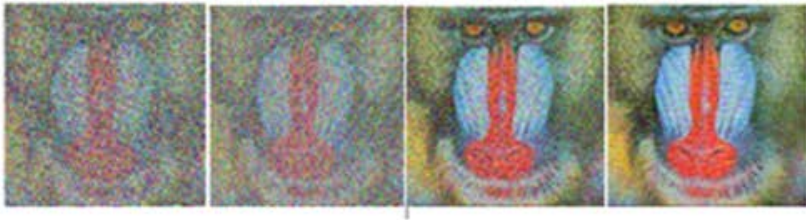

(a) $\mathrm{nTx}=1$ and $\mathrm{nRx}=1$
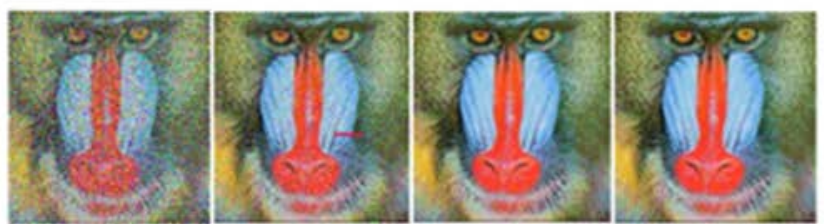

(b) $n T x=1$ and $n R x=2$.

Figure 9. Received Image with DCT-OFDM over a Rayleigh channel, Modulated with QPSK at Eb/No = 0, 5, 10, 15 dB from left to right with MRC (nTx = 1 and $n R x=1$ and 2 ).

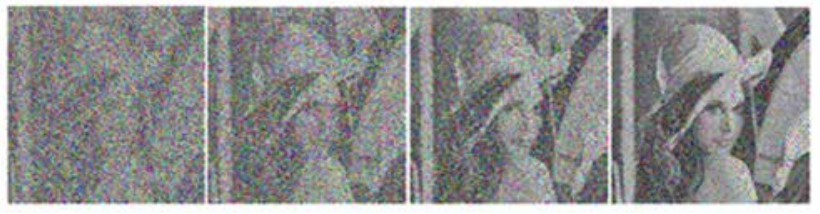

(a) $n T x=1$ and $n R x=1$

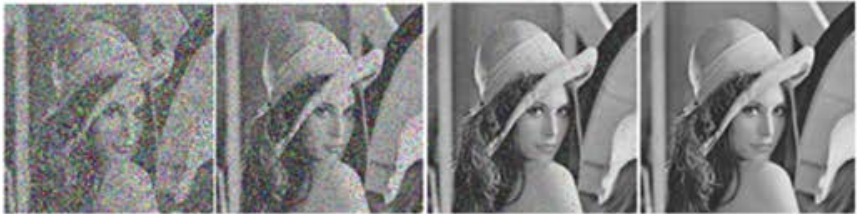

(b) $n T x=1$ and $n R x=2$.

Figure 10. Received Image with FFT-OFDM over a Rayleigh channel, Modulated with QPSK at Eb/No = 0, 5, 10, $15 \mathrm{~dB}$ from left to right with MRC ( $\mathrm{nTx}=1$ and $\mathrm{nRx}=1$ and 2). 


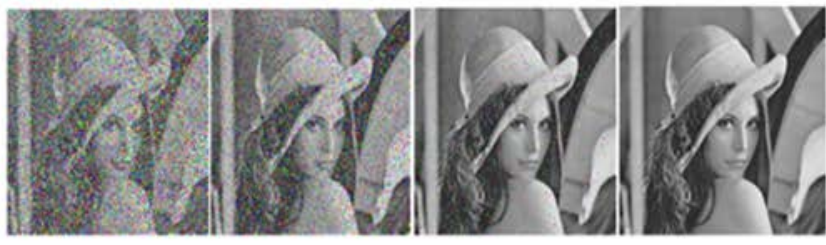

(a) $\mathrm{nTx}=1$ and $\mathrm{nRx}=1$

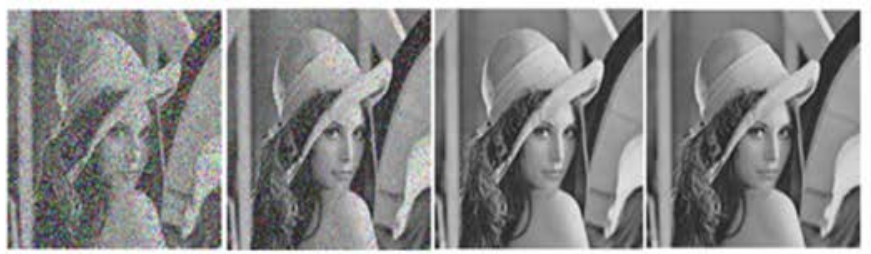

(b) $n T x=1$ and $n R x=2$.

Figure 11. Received Image with DCT-OFDM over a Rayleigh channel, Modulated with QPSK at Eb/No = 0, 5, 10, $15 \mathrm{~dB}$ from left to right with MRC ( $\mathrm{nTx}=1$ and $\mathrm{nRx}=1$ and 2).

Table 10 presents the analysis of PSNR and SSIM variations w.r.t SNR, number of receiving antenna and transform, i.e., FFT/DCT for OFDM system for the RGB image (Baboon), and Table 11 presents the analysis of PSNR and SSIM variations w.r.t SNR, number of receiving antenna and transform, i.e., FFT/DCT for OFDM system for the grayscale image (Lena). It is quite evident from Tables 10 and 11 that the PSNR and SSIM value is improving in implementing the DCT in place of FFT. Further improvement is also there on incorporating the MRC technique.

Table 10. PSNR and SSIM variations for FFT-OFDM and DCT-OFDM for QPSK with MRC (nTx = 1 and $n R x=1,2,3)$ over Rayleigh Channel for RGB image (Baboon).

\begin{tabular}{|c|c|c|c|c|c|c|c|c|c|}
\hline \multirow{2}{*}{ System } & \multirow{2}{*}{$\begin{array}{c}\text { Antenna } \\
\text { Configuration }\end{array}$} & \multicolumn{2}{|c|}{$\mathrm{SNR}=0 \mathrm{~dB}$} & \multicolumn{2}{|c|}{$\mathrm{SNR}=5 \mathrm{~dB}$} & \multicolumn{2}{|c|}{$\mathrm{SNR}=10 \mathrm{~dB}$} & \multicolumn{2}{|c|}{$\mathrm{SNR}=15 \mathrm{~dB}$} \\
\hline & & PSNR & SSIM & PSNR & SSIM & PSNR & SSIM & PSNR & SSIM \\
\hline \multirow{3}{*}{$\begin{array}{c}\text { FFT- } \\
\text { OFDM }\end{array}$} & $1 \mathrm{Tx}$ and $1 \mathrm{Rx}$ & 9.76543 & 0.03915 & 10.705663 & 0.07199 & 11.58676 & 0.111477 & 15.3062 & 0.2962 \\
\hline & $1 \mathrm{Tx}$ and $2 \mathrm{Rx}$ & 11.9468 & 0.12827 & 15.321285 & 0.3044454 & 23.93093 & 0.775219 & 51.2156 & 0.9994 \\
\hline & 1 Tx and $3 \mathrm{Rx}$ & 13.2219 & 0.1910 & 18.5414 & 0.4963 & 32.5573 & 0.9624 & 73.2458 & 1.0000 \\
\hline \multirow{3}{*}{$\begin{array}{l}\text { DCT- } \\
\text { OFDM }\end{array}$} & $1 \mathrm{Tx}$ and $1 \mathrm{Rx}$ & 9.67864 & 0.04332 & 9.6975001 & 0.0227244 & 14.11069 & 0.236522 & 20.5423 & 0.6079 \\
\hline & $1 \mathrm{Tx}$ and $2 \mathrm{Rx}$ & 13.5783 & 0.2069 & 19.38939 & 0.545288 & 35.45733 & 0.980889 & 78.4622 & 1 \\
\hline & $1 \mathrm{Tx}$ and $3 \mathrm{Rx}$ & 15.8745 & 0.3314 & 25.2924 & 0.8255 & 56.8592 & 0.9998 & 87.5695 & 1.0000 \\
\hline
\end{tabular}

Table 11. PSNR and SSIM variations for FFT-OFDM and DCT-OFDM for QPSK with MRC (nTx = 1 and nRx $=1,2,3)$ over Rayleigh Channel for grayscale image (Lena).

\begin{tabular}{|c|c|c|c|c|c|c|c|c|c|}
\hline \multirow{2}{*}{ System } & \multirow{2}{*}{$\begin{array}{c}\text { Antenna } \\
\text { Configuration }\end{array}$} & \multicolumn{2}{|c|}{$\mathrm{SNR}=0 \mathrm{~dB}$} & \multicolumn{2}{|c|}{$\mathrm{SNR}=5 \mathrm{~dB}$} & \multicolumn{2}{|c|}{$\mathrm{SNR}=10 \mathrm{~dB}$} & \multicolumn{2}{|c|}{$\mathrm{SNR}=15 \mathrm{~dB}$} \\
\hline & & PSNR & SSIM & PSNR & SSIM & PSNR & SSIM & PSNR & SSIM \\
\hline \multirow{3}{*}{$\begin{array}{l}\text { FFT- } \\
\text { OFDM }\end{array}$} & $1 \mathrm{Tx}$ and $1 \mathrm{Rx}$ & 9.89905 & 0.0293 & 10.592833 & 0.0430585 & 11.81925 & 0.085431 & 15.504 & 0.21941 \\
\hline & $1 \mathrm{Tx}$ and $2 \mathrm{Rx}$ & 12.0372 & 0.0938 & 15.224939 & 0.211088 & 23.72912 & 0.646574 & 48.3271 & 0.99792 \\
\hline & $1 \mathrm{Tx}$ and $3 \mathrm{Rx}$ & 13.2472 & 0.1336 & 18.4498 & 0.3581 & 32.2927 & 0.9355 & 72.4842 & 1.0000 \\
\hline \multirow{3}{*}{$\begin{array}{c}\text { DCT- } \\
\text { OFDM }\end{array}$} & $1 \mathrm{Tx}$ and $1 \mathrm{Rx}$ & 10.2949 & 0.0411 & 11.182142 & 0.0651304 & 12.34516 & 0.119801 & 18.1675 & 0.33916 \\
\hline & 1 Tx and 2 Rx & 13.5942 & 0.1467 & 19.413126 & 0.4074441 & 35.84002 & 0.964057 & 78.6842 & 1 \\
\hline & 1 Tx and $3 \mathrm{Rx}$ & 15.8437 & 0.2359 & 25.1688 & 0.7168 & 48.3627 & 0.9988 & 85.9384 & 1.0000 \\
\hline
\end{tabular}

The SNR vs. BER performance analysis of MRC augmented FFT-OFDM and DCTOFDM is presented over AWGN and Rayleigh channels in Figures 12 and 13. The performance of both the FFT-OFDM and DCT-OFDM improves significantly on using the MRC schemes employing multiple antennas at the receiver end. 


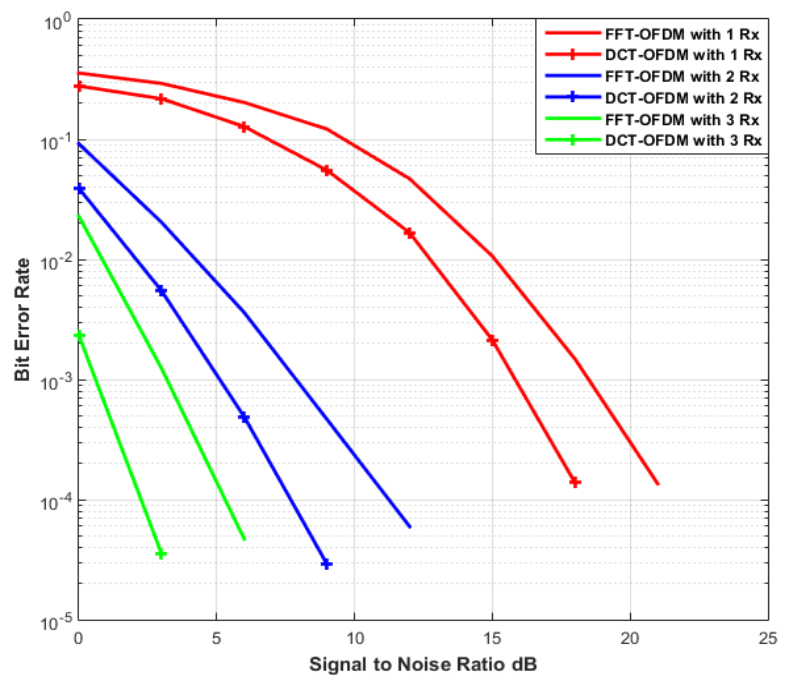

(a)

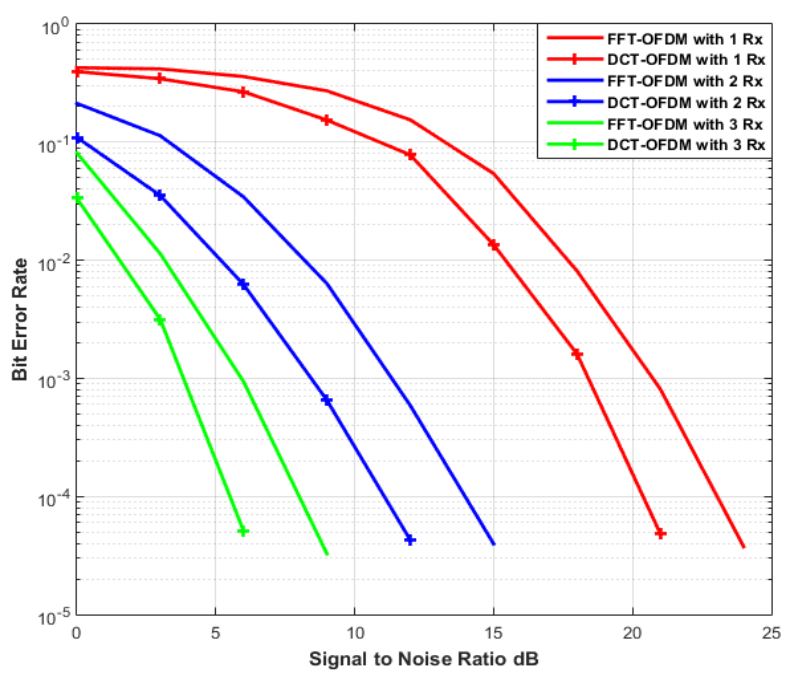

(b)

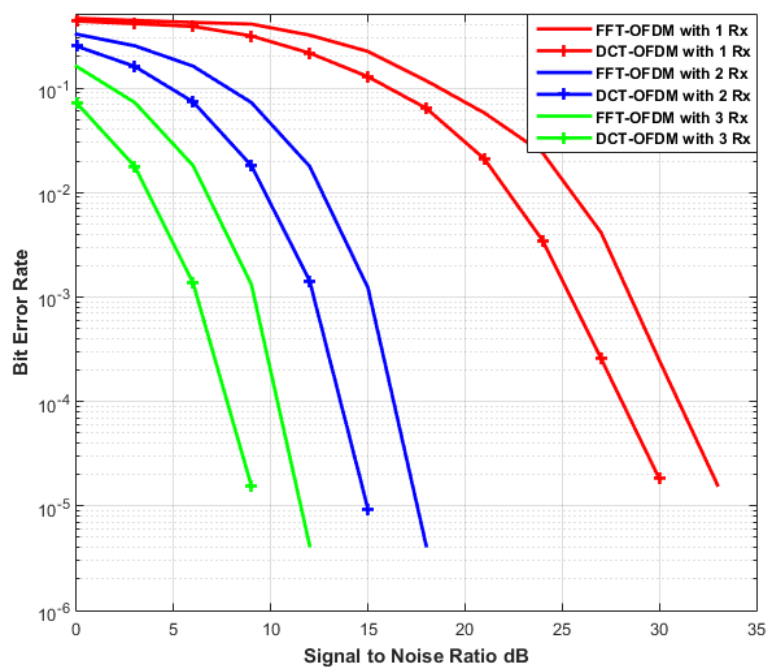

(c)

Figure 12. Cont. 


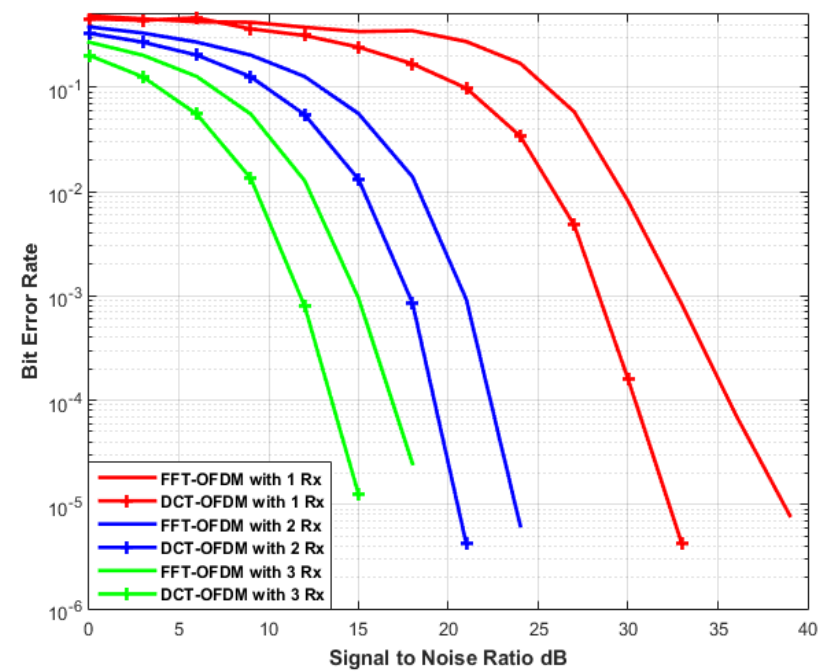

(d)

Figure 12. SNR vs. BER comparison of FFT-OFDM and DCT-OFDM for diverse Modulations over AWGN channel with MRC ( $\mathrm{nTx}=1$ and $\mathrm{nRx}=1,2,3)$, (a) BPSK (b) QPSK (c) 8-PSK (d) 16-PSK.

As illustrated by Figure 12a, to achieve a BER of $10^{-4}$, in AWGN channel scenario, BPSK modulated FFT-OFDM necessitates SNR of $19 \mathrm{~dB}$, but it reduces up to $8 \mathrm{~dB}$ for FFT-OFDM with MRC ( 1 Tx and 2 and $3 \mathrm{Rx}$ ). A similar observation can be made for the DCT-OFDM with MRC ( $1 \mathrm{Tx}$ and 2 and $3 \mathrm{Rx}$ ), which requires $5.3 \mathrm{~dB}$ of SNR in comparison to $16 \mathrm{~dB}$ of SNR it requires without MRC. For QPSK modulation in AWGN scenario, FFT-OFDM requires $23.5 \mathrm{~dB}$ of SNR, which is more than $12 \mathrm{~dB}$ required in the case of FFT-OFDM with MRC. On the other hand, the same modulation system requires $22 \mathrm{~dB}$ and $10.5 \mathrm{~dB}$ of SNR to achieve the desired BER for DCT-OFDM without MRC and DCT-OFDM with MRC. Consequently, it is summarized that DCT-OFDM improves 1-3 dB of SNR over FFT-OFDM in AWGN and Rayleigh fading channel scenarios. It is further concluded that on employing the MRC schemes, significant improvement in SNR ranging in between 10-12 $\mathrm{dB}$ for both FFT-OFDM and DCT-OFDM has been reported in work.

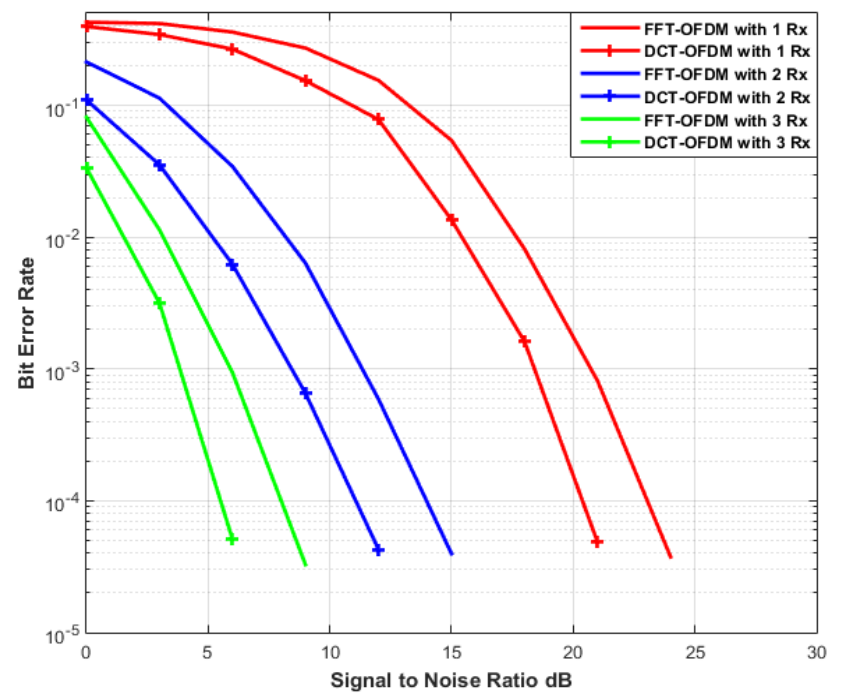

(a)

Figure 13. Cont. 


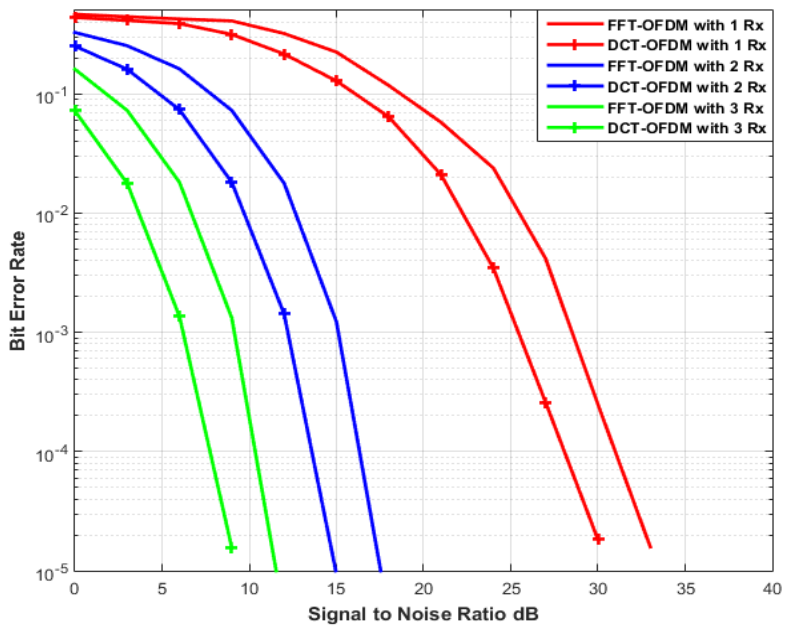

(b)

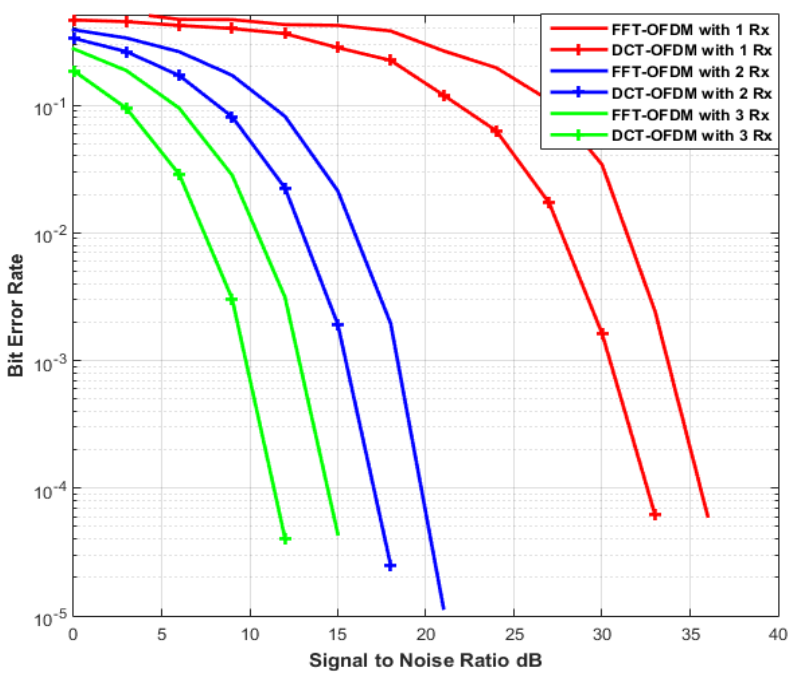

(c)

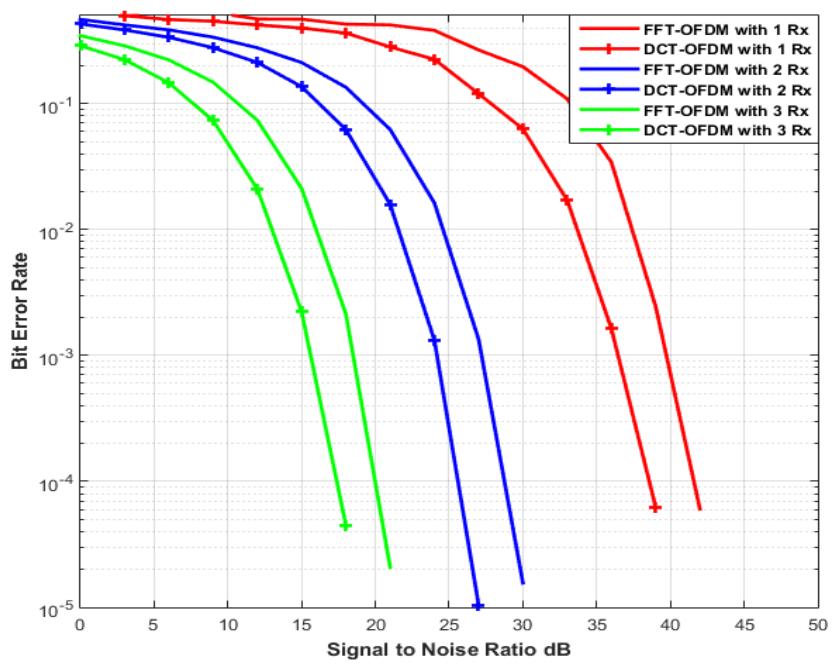

(d)

Figure 13. SNR vs. BER comparison of FFT-OFDM and DCT-OFDM for diverse Modulations over Rayleigh channel with MRC (nTx = 1 and $\mathrm{nRx}=1,2)$, (a) BPSK (b) QPSK (c) 8-PSK (d) 16-PSK. 
To emphasize that increase in BER will lead to a decrease in data rate, as depicted in Figures 12 and 13, as we go on increasing the modulation level to elevate the data rate the BER also keeps on increasing. This increase of BER will have adverse effects on the quality of the image being received at the receiver end, which can only be compensated by an increase in SNR. As the increase in SNR will result in increased signal strength at the receiver end, which will further augment the signal demodulation process at the receiver side. The position of constellation points for the received symbols at the input of demodulator will move to the ideal position on the constellation diagram, which will result in correct decision making at the output of the demodulator, and BER will decrease.

However, in the modern-day systems, we cannot increase the SNR that easily as it may lead to increase size and cost of the equipment. These constraints will restrict the system operation to lower the modulation level, which will result in a reduction in data rate. Hence, the reduction in BER will allow us to switch to higher modulation levels and achieving higher data rates by keeping the SNR requirement in desirable limits and making the proposed system a suitable contender for $5 \mathrm{G}$ applications in smart cities. Moreover, the reliable mode of image communication will efficiently address the current need of diverse user drive application such as real-time monitoring of roads, smart homes, smart industries, crop disease monitoring, crop growth monitoring, surface defect detection, etc. [38,39].

\section{Conclusions and Future Scope}

In this paper, the concept of robust and efficient image transmission required for a sustainable smart city is realized by utilizing a hybrid combination of MIMO and OFDM systems based on two discrete transforms, i.e., FFT and DCT. The simulation results on images show a significant improvement in BER performance for both the transform when the number of receivers is increased from 1 to 2 , making it a more sustainable model by decreasing the requirement of high SNR. The improvement goes up to $10 \mathrm{~dB}$ in terms of SNR required on switching from 1 to 2 receiving antennas. The image quality is also improving significantly, which can be accessed by visual inspection and the PSNR and SSIM values, which ensures efficient implementation of real-time monitoring methodologies critical for the smart city concept. Further, it can be observed that DCT based OFDM system performs better than FFT based OFDM. The BER improvement provided by DCT based OFDM is going up to $3 \mathrm{~dB}$ regarding SNR required. The same effect can be seen on the image quality also, where the PSNR and SSIM values are even more in the case of DCT based OFDM in comparison to FFT-based OFDM. Additionally, the performance of the proposed methodology over AWGN channel is better than the Rayleigh fading channel. OFDM systems augmented with MIMO provide better performance as we keep on increasing the number of antennas. Moreover, the proposed methodology can be used to transmit multimedia information, thereby making it a better contender for smart city applications.

In the future, this work can be extended for a hybrid system of OFDM and massive MIMO to support the multimedia application for 5G users. This work can also be used to incorporate and study diverse transformation techniques instead of conventional Fourier transform to make the system more robust and efficient.

Author Contributions: Conceptualization, L.K., G.S.G.; Data curation, L.K., G.S.G.; Formal analysis, L.K., N.C.; Investigation, L.K., N.C.; Methodology, L.K., G.S.G., N.C. and B.-G.K.; Resources, B.-G.K.; Visualization, L.K.; Writing—original draft, L.K., G.S.G.; Writing—review \& editing, L.K., G.S.G., N.C. and B.-G.K. All authors have read and agreed to the published version of the manuscript.

Funding: This research received no funding.

Informed Consent Statement: Not applicable.

Data Availability Statement: The datasets generated and/or analysed during this study are available from the corresponding author on reasonable request.

Conflicts of Interest: The authors declare no conflict of interest. 


\section{References}

1. Banelli, P.; Colavolpe, G.; Rugini, L.; Ugolini, A. Spectral Efficiency of Multicarrier Schemes for 5G. In Proceedings of the 2019 26th International Conference on Telecommunications (ICT), Hanoi, Vietnam, 8-10 April 2019; pp. 124-129.

2. Foschini, G.J.; Salz, J. Digital communications over fading radio channels. Bell Syst. Tech. J. 1983, 62, 429-456. [CrossRef]

3. Brennan, D.G. Linear diversity combining techniques. Proc. IEEE 2003, 91, 331-356. [CrossRef]

4. Gans, M. The effect of Gaussian error in maximal ratio combiners. IEEE Trans. Commun. Technol. 1971, 19, 492-500. [CrossRef]

5. Chyi, G.T.; Proakis, J.G.; Keller, C.M. On the symbol error probability of maximum-selection diversity reception schemes over a Rayleigh fading channel. IEEE Trans. Commun. 1989, 37, 79-83. [CrossRef]

6. Winters, J.H.; Salz, J.; Gitlin, R.D. The impact of antenna diversity on the capacity of wireless communication systems. IEEE Trans. Commun. 1994, 42, 1740-1751. [CrossRef]

7. Wan, Y.; Chen, J.C. Fading distribution of diversity techniques with correlated channels. In Proceedings of the International Symposium on Personal, Indoor and Mobile Radio Communications, Toronto, ON, Canada, 27-29 September 1995; pp. $1202-1206$.

8. Cui, J.; Falconer, D.D.; Sheikh, A.U.H. The analysis of BER for opti-mum combining with two co-channel interferers and maximal ratio combining with arbitrary number of interferers. In Proceedings of the International Symposium on Personal, Indoor and Mobile Radio Communications, Taipei, Taiwan, 18 October 1996; pp. 53-57.

9. Zhang, Q.T. Probability of error for equal-gain combiners over Rayleigh channels: Some closed-form solutions. IEEE Trans. Commun. 1997, 45, 270-273. [CrossRef]

10. Cui, J.; Falconer, D.D.; Sheikh, A.U.H. BER analysis of optimum combining and maximal ratio combining with channel correlation for dual antenna systems. In Proceedings of the Vehicular Technology Conference, Phoenix, AZ, USA, 4-7 May 1997; pp. 150-154

11. Lo, T.K. Maximum ratio transmission. In Proceedings of the 1999 IEEE International Conference on Communications, Vancouver, BC, Canada, 6-10 June 1999; pp. 1310-1314.

12. Win, M.Z.; Winters, J.H. Analysis of hybrid selection/maximal-ratio combining in Rayleigh fading. In Proceedings of the IEEE Conference on Communication, Vancouver, BC, Canada, 6-10 June 1999; pp. 1773-1776.

13. Thoen, S.; Van der Perre, L.; Gyselinckx, B.; Engels, M. Performance analysis of combined transmit-SC/receive-MRC. IEEE Trans. Commun. 2001, 49, 5-8. [CrossRef]

14. Win, M.Z.; Winters, J.H. Virtual branch analysis of symbol error probability for hybrid selection/maximal-ratio combining in Rayleigh fading. IEEE Trans. Commun. 2001, 49, 1926-1934. [CrossRef]

15. Molisch, A.F.; Win, M.Z.; Winters, J.H. Reduced-complexity transmit/receive-diversity systems. IEEE Trans. Signal Process. 2003, 51, 2729-2738. [CrossRef]

16. Chen, Z.; Yuan, J.; Vucetic, B. Analysis of transmit antenna selection/maximal-ratio combining in Rayleigh fading channels. IEEE Trans. Veh. Technol. 2005, 54, 1312-1321. [CrossRef]

17. Tang, J.; Zhang, X. Transmit selection diversity with maximal-ratio combining for multicarrier DS-CDMA wireless networks over Nakagami-m fading channels. IEEE J. Sel. Areas Commun. 2005, 24, 104-112. [CrossRef]

18. Wang, B. Accurate BER of transmitter antenna selection/receiver-MRC over arbitrarily correlated Nakagami fading channels. In Proceedings of the IEEE International Conference on Acoustics Speech and Signal Processing, Toulouse, France, 14-19 May 2006; pp. 753-756.

19. Kang, M.; Alouini, M.S. Performance Analysis of MIMO MRC Systems over Rician Fading Channels. In Proceedings of the IEEE Vehicular Technology Conference, Vancouver, BC, Canada, 24-28 September 2002; pp. 869-873.

20. Cheng, J.; Berger, T. Performance analysis for MRC and post defection EGC over generalized gamma fading channels. In Proceedings of the IEEE Wireless Communication and Networking Conference, New Orleans, LA, USA, 16-20 March 2003; pp. 120-125.

21. Al-Dhahir, N.; Minn, H. A new multicarrier transceiver based on the discrete cosine transform. In Proceedings of the IEEE Wireless Communications and Networking Conference, New Orleans, LA, USA, 13-17 March 2005; pp. 45-50.

22. Aalo, V.A.; Piboongungon, T.; Efthymoglou, G.P. Another look at the performance of MRC schemes in Nakagami-m fading channels with arbitrary parameters. IEEE Trans. Commun. 2005, 53, 2002-2005. [CrossRef]

23. Tan, P.; Beaulieu, N.C. Precise bit error probability analysis of DCT OFDM in the presence of carrier frequency offset on AWGN channels. In Proceedings of the IEEE Global Telecommunication Conference, St. Louis, MO, USA, 28 November-2 December 2005; pp. 1429-1434.

24. Tan, P.; Beaulieu, N.C. A comparison of DCT-based OFDM and DFT-based OFDM in frequency offset and fading channels. IEEE Trans. Commun. 2006, 54, 2113-2125. [CrossRef]

25. Tan, P.; Beaulieu, N.C. An improved DCT-based OFDM data transmission scheme. In Proceedings of the International Symposium on Personal, Indoor and Mobile Radio Communications, Berlin, Germany, 11-14 September 2005; pp. 745-749.

26. Gupta, D.; Vats, V.B.; Garg, K.K. Performance analysis of DFT-OFDM, DCT-OFDM, and DWT-OFDM systems in AWGN. In Proceedings of the IEEE 4th International Conference on Wireless and Mobile Communications, Athens, Greece, 27 July-1 August 2008; pp. 214-216.

27. Lin, S.C. Performance analysis for optimum transmission and comparison with maximal ratio transmission for MIMO systems with cochannel interference. EURASIP J. Wirel. Commun. Netw. 2011, 2011, 89. [CrossRef]

28. Ullah, M.S.; Uddin, M.J. Performance analysis of wireless MIMO system by using Alamouti's scheme and maximum ratio combining technique. Int. J. Adv. Eng. Sci. Technol. 2011, 8, 19-24. 
29. Eldin, S.M.S. Optimized OFDM transmission of encrypted image over fading channel. Sens. Imaging 2014, 15, 99. [CrossRef]

30. Soliman, N.F.; Hassan, E.S.; Shaalan, A.H.A.; Fouad, M.M.; El-Khamy, S.E.; Albagory, Y.; El-Bendary, M.A.; Al-Hanafy, W.; El-Rabaie, E.S.M.; Dessouky, M.I.; et al. Efficient image communication in PAPR distortion cases. Wirel. Pers. Commun. 2015, 83, 2773-2834. [CrossRef]

31. Kansal, L.; Sharma, V.; Singh, J. Performance assessment of precoded OFDM using discrete cosine-based DOST transform. Optik 2016, 127, 7242-7249. [CrossRef]

32. Kansal, L.; Sharma, V.; Singh, J. BER assessment of FFT-OFDM against WHT-OFDM over different fading channel. Wirel. Netw. 2017, 23, 2189-2196. [CrossRef]

33. Wang, Z.; Chen, F.; Qiu, W.; Chen, S.; Ren, D. A two layer chaotic encryption scheme of secure image transmission for DCT precoded OFDM-VLC transmission. Opt. Commun. 2018, 410, 94-101. [CrossRef]

34. Agarwal, A.; Kumar, B.S.; Agarwal, K. BER Performance Analysis of Image Transmission Using OFDM Technique in Different Channel Conditions Using Various Modulation Techniques. In Computational Intelligence in Data Mining; Advances in Intelligent Systems and Computing; Springer: Singapore, 2018; pp. 1-8.

35. Khaliluzzaman, M.; Pervin, S.; Ima, U.M.; Chy, D.K. DWT and DCT-Based Compressed Image Transmission Over AWGN Using C-QAM. In Emerging Technologies in Data Mining and Information Security; Advances in Intelligent Systems and Computing; Springer: Singapore, 2018; pp. 337-351.

36. Rammyaa, B.; Vishvaksenan, K.S.; Poobal, S. Performance of spectrum sharing cognitive radio network based on MIMO MC-CDMA system for medical image transmission. Clust. Comput. 2019, 22, 7705-7712. [CrossRef]

37. Kansal, L.; Sharma, V.; Singh, J. Multiuser Massive MIMO-OFDM System Incorporated with Diverse Transformation for 5G Applications. Wirel. Pers. Commun. 2019, 109, 2741-2756. [CrossRef]

38. Yang, J.; Wang, C.; Jiang, B.; Song, H.; Meng, Q. Visual Perception Enabled Industry Intelligence: State of the Art, Challenges and Prospects. IEEE Trans. Ind. Inform. 2020, 17, 2204-2219. [CrossRef]

39. Wen, J.; Yang, J.; Jiang, B.; Song, H.; Wang, H. Big Data Driven Marine Environment Information Forecasting: A Time Series Prediction Network. IEEE Trans. Fuzzy Syst. 2020, 29, 4-18. [CrossRef] 